\title{
Relationship between preoperative anxiety and postoperative satisfaction in dental implant surgery with intravenous conscious sedation
}

\author{
Sandra González-Lemonnier ${ }^{1}$, Maite Bovaira-Forner ${ }^{2}$, María Peñarrocha-Diago ${ }^{3}$, David Peñarrocha-Oltra ${ }^{1}$
}

\author{
${ }^{1}$ Resident of the Master of Oral Surgery and Implantology. Valencia University Medical and Dental School. Valencia \\ ${ }^{2}$ Specialist in Anesthesiology and Resuscitation. Levante Rehabilitation Center. Valencia \\ ${ }^{3}$ Associated Professor of Oral Surgery. Valencia University Medical and Dental School
}

Correspondence:

Facultad de Medicina y Odontología

Gascó Oliag 1

46021 - Valencia (Spain)

miguel.penarrocha@uv.es

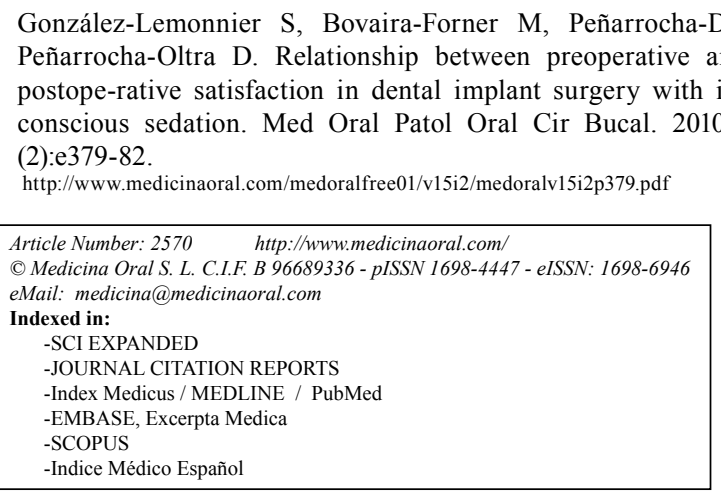

\begin{abstract}
Purpose: To study if patient preoperative anxiety is related to age and gender and to compare preoperative anxiety with postoperative patient and surgeon satisfaction in dental implant surgery under intravenous conscious sedation. Materials and Methods: Dental implants were placed in 102 patients under local anesthesia and intravenous conscious sedation. The procedures were performed with or without dental extractions, and with or without bone regeneration. Anxiety was evaluated using Corah's Dental Anxiety Scale and levels of surgeon and patient satisfaction were evaluated on an adapted scale. Results: Low preoperative anxiety was observed in $27.8 \%$ of patients, moderate in $50 \%$, and high in $22.2 \%$. Mean value of anxiety was $9.8+/-3.7$. The level of surgeon satisfaction was adequate in $87.8 \%$ of the surgeries; patients were awake and nervous in $4.4 \%$ of surgeries, and excessively sleepy, with little cooperation in $7.8 \%$ of surgeries. Regarding patient satisfaction, the procedure was comfortable for $23.3 \%$ of patients, neither comfortable nor uncomfortable for $28.9 \%$, a slightly uncomfortable experience for $36.7 \%$, and very uncomfortable for $10 \%$ of patients. Younger patients and women were observed to have more anxiety, the difference being statistically significant. Patients with higher preoperative anxiety expressed a lower level of satisfaction, with statistically significant differences. There was no significant relationship between preoperative patient anxiety and postoperative surgeon satisfaction. Conclusion: Anxiety was higher in younger patients and women. In this study, a higher preoperative patient anxiety was associated with lower patient satisfaction, but had no influence on postoperative surgeon satisfaction.
\end{abstract}

Key words: Anxiety, satisfaction, dental implants. 


\section{Introduction}

Anxiety is an emotional reaction defined as tension (stress), apprehension, nervousness and concern caused because by an intangible and diffuse advancing threat or approaching danger, accompanied by activation the autonomous nervous system (1). Anxious patients are uncooperative during implant surgery and complicate the procedure (2). In dental implant surgery, where a very long procedure is expected, local anesthesia can be insufficient to perform an adequate operation, and conscious intravenous sedation is an option (3). In conscious intravenous sedation the lowering of consciousness is minimal, the patient maintains verbal communication and cooperation, vital signs are stable, the airway is unobstructed and active reflex protectors are maintained (4). Conscious intravenous sedation with local anesthesia is a safe alternative to general anesthesia for the control of intraoperative pain and anxiety in oral surgery procedures (5).

Given the growing demand for conscious intravenous sedation for oral surgery, it is interesting to know the grade of patient anxiety in dental implant surgery under conscious intravenous sedation. The aim was to study whether preoperative anxiety of the patient is influenced by age and sex and to compare the preoperative anxiety of the patient with the postoperative patient and surgeon satisfaction in dental implant surgery with local anesthesia and conscious intravenous sedation.

\section{Material and Methods}

Between May 2004 and February 2007, 102 patients were treated with dental implants under local anesthesia and conscious intravenous sedation. Indication for conscious intravenous sedation was the placing of 4 or more implants, with or without dental extractions, and with or without bone regeneration, or if the surgical procedure was to last for more than 60 minutes. Exclusion criteria were incomplete questionnaires returned by patients. Patients were instructed to fast for 6 hours before surgery and to bring a responsible person to accompany them home after sedation. All patients were treated in a clinic equipped with the appropriate sedation and monitoring facilities.

Before surgery, in the waiting room, patients completed a questionnaire to evaluate preoperative dental anxiety by means of Corah's scale (Fig. 1) (6). The questionnaire contains 4 items about different feelings concerning dental consultation. Each question has 5 possible answers, ranging from a) $=1$ (no anxiety) to e) $=5$ (high anxiety). Therefore, the possible score ranges from 4 to 20. Anxiety is considered low when scores are equal to or less than 6 , moderate with scores between 7 and 12 , and high with scores equal to or greater than 13 (7). The procedure was carried out by the same surgeon and anesthetist. The protocol for administration of conscious
1. If you had to go to the dentist tomorrow, how would you feel about it?

a) I would look forward to it as a reasonably enjoyable experience

b) I wouldn't care one way or the other

c) I would be a little uneasy about it

d) I would be afraid that it would be unpleasant and painful

e) I would be very frightened of what the dentist might do

2 . When you are waiting in the dentist's office for your turn in the chair, how do you feel?

a) Relaxed

b) A little uneasy

c) Tense

d) Anxious

e) So anxious that I sometimes break out in a sweat or almost feel physically sick

3. When you are in the dentist's chair waiting while he gets his drill ready to begin working on your teeth, how do you feel?

a) Relaxed

b) A little uneasy

c) Tense

d) Anxious

e) So anxious that I sometimes break out in a sweat or almost feel physically sick

1. You are in the dentist's chair to have your teeth cleaned. While you are waiting and the dentist is getting out the instruments which he will use to scrape your teeth around the gums, how do you feel?

a) Relaxed

b) little uneasy

c) Tense

d) Anxious

e) So anxious that I sometimes break out in a sweat or almost feel physically sick

Fig. 1. Corah's dental anxiety scale.

sedation was: midazolam $0.05 \mathrm{mg} / \mathrm{kg}$ and fentanyl $1 \mu \mathrm{g} /$ $\mathrm{kg}$; if good sedation was achieved, the local anesthesia was injected. Articaine 4\% with adrenaline 1:200.000 was used under normal conditions, or when vasoconstrictor was contraindicated we used mepivacaine without vasoconstrictor. If the patient remained tense propofol 20-30 mg was added.

Anesthesia was maintained with midazolam $1 \mathrm{mg}$ and fentanyl $0.5-1 \mu \mathrm{g} / \mathrm{kg}$, every 30 or 60 minutes in function of patient state and anesthetic depth. If necessary, propofol was administered on demand in successive bolus of $20 \mathrm{mg}$. Atropine $0.01 \mathrm{mg} / \mathrm{kg}$ was administered to patients under 30 years of age or with a basal heart rate lower than $60 \mathrm{bpm}$ when no contraindicating medication was present.

Levels of surgeon satisfaction were recorded immediately after surgery in 3 degrees: a) too awake and nervous; b) adequate level of sedation; and c) excessively sleepy, no cooperation. Patient satisfaction was recorded on 5 levels: a) agreeable experience; b) neither pleasant nor unpleasant; c) slightly uncomfortable; d) disagreeable; e) a traumatic experience.

Descriptive data of anxiety and level of surgeon and patient satisfaction were analyzed. To study the relationship between anxiety and satisfaction the Spearman 
correlation coefficient was used. To study the relationship between anxiety and age the Pearson correlation was used. Differences in anxiety between man and women were studied by the student's $t$ test. Statistical significance was considered for $\mathrm{p}$ values less than .05 in all cases.

\section{Results}

Ninety patients undergoing implant surgery with conscious intravenous sedation were included in this study. Twelve were excluded for returning incomplete questionnaires. A total of 693 dental implants were placed (average 7.7 implants per patient). Average time of surgical procedure was 98 minutes. Dental implants without dental extraction and bone regeneration were placed in 53 patients; postextraction implants were placed in 24 patients; implants with bone regeneration in 10 patients, and postextraction implants with bone regeneration in 3 patients.

Preoperative anxiety was low in $27.8 \%$; moderate in $50 \%$, and high in $22.2 \%$. Mean value of preoperative anxiety was $9.8 \pm 3.7$. Younger patients showed more preoperative anxiety, the differences being statistically significant $(r=-0.285 ; p=0.006)$. Anxiety was higher in women, the differences being statistically significant $(\mathrm{t}=2.6 ; \mathrm{p}=0.01)$.

The level of satisfaction for the surgeon was adequate in $87.8 \%$ of surgeries, in $4.4 \%$ the surgeon considered the patient "too awake and nervous" and in $7.8 \%$, that the patient was "excessively sleepy, no cooperation".

The experience was agreeable for $23.3 \%$ of patients, neither pleasant nor unpleasant for $28.9 \%$, slightly uncomfortable for $36.7 \%$, and a disagreeable experience for $10 \%$ of patients.

A higher preoperative patient anxiety was associated with lower patient satisfaction $(\mathrm{r}=0.296 ; \mathrm{p}=0.005)$. There was no significant relationship between preoperative anxiety and postoperative surgeon satisfaction (Table 1).

Table 1. Relationship between preoperative anxiety with age, sex and postoperative satisfaction.

\begin{tabular}{|l|c|c|}
\hline & \multicolumn{2}{|c|}{ Preoperative anxiety } \\
\hline & Statistical test & $\mathrm{P}$ \\
\hline Age & $\mathrm{r}_{1}=-0.285$ & $\mathrm{p}=0.006$ \\
\hline Sex & $\mathrm{t}=2.6$ & $\mathrm{p}=0.01$ \\
\hline Postoperative satisfaction & $\mathrm{r}_{2}=0.296$ & $\mathrm{p}=0.005$ \\
\hline
\end{tabular}

r1: Pearson correlation; t: student's t test; r2: Spearman correla tion; p: statistical significance.

\section{Discussion}

Placement of dental implants is one of the most anxiety-provoking oral surgery procedures (8). In a study by Ellis (9), where patients were intravenously administered midazolam in third molar surgery, $49 \%$ had high anxiety and showed more intraoperative movements and less cooperation. On the other hand, using Corah's scale, Parworth et al. (10) found no statistically significant differences between propofol with fentanyl and midazolam with fentanyl, administered intravenously in third molar surgery. In our study, $72.2 \%$ of patients had between moderate and high levels of anxiety during the dental implant placement. The mean value of Corah's scale in our study was $9.8 \pm 3$.7. These results are higher than previous studies: in Germany (11), the mean was $8.6 \pm 3.7$ (11) and in Norway it was 7.9 \pm 3.5 (12).

Liddell and Locker, in their study, found that preoperative anxiety decreased with age (13). Hägglin et al. (14) explained that technological advances in dentistry can decrease this anxiety. Liau et al. (15), found that younger patients had higher anxiety, arguing that experience and familiarity are important factors. In the present study, in agreement with previous authors, had the difference statistically significant between age and preoperative anxiety, being less the anxiety with more age. Sex of patients is one of the most analyzed variables in studies of dental anxiety. Hashem et al. (16) found no significant differences in anxiety between women and men. Our study agrees with other authors (17), in that women had higher anxiety than men.

Ganzberg et al. (18) obtained a mean of $85.8 \%$ surgeon satisfaction (evaluated on a 0 to 100 visual analogue scale) using conscious intravenous sedation in third molar surgery. Garip et al (19), using conscious intravenous sedation in third molar surgery, found that surgeon satisfaction was excellent in 55\% of procedures (evaluated as: unacceptable, poor, satisfactory, good and excellent). In the present study, the surgeon considered the level of sedation to have been adequate in $87.8 \%$ of procedures. In the present study, the level of patient satisfaction at the end of dental implant surgery with conscious intravenous sedation was between agreeable and neither pleasant nor unpleasant for $52.2 \%$ of patients, and for $36.7 \%$ slightly uncomfortable. These results differ from Ganzberg et al. (18) who obtained a mean of $84.1 \%$ patient satisfaction (evaluated on a visual analogue scale, from 0 to 100), and Garip et al. (19) who found that $95 \%$ of patients expressed excellent satisfaction (evaluated as excellent, good and poor).

In our study, the surgeon carried out implant placement under conscious intravenous sedation with adequate satisfaction, showing the patients relaxed and collaborative with the dentist. The anesthetist monitors the surgical-sedative procedure and the patient's state of mind, so that the surgeon can concentrate on the surgery. In 
these cases, conscious intravenous sedation was used in implantology, being of interest in other fields of oral surgery.

Preoperative anxiety was higher in younger and women. A higher preoperative patient anxiety was associated with lower satisfaction, but had no influence on postoperative surgeon satisfaction.

\section{References}

1. Miller AA. Psychological considerations in dentistry. J Am Dent Assoc. 1970;81:941-8.

2. Smyth JS. Some problems of dental treatment. Part 1. Patient anxiety: some correlates and sex differences. Aust Dent J. 1993;38:354-9.

3. Chanavaz M, Ferri J, Donazzan M. Intravenous sedation in implantology. Rev Stomatol Chir Maxillofac. 1997;98:57-61.

4. Bennett CR. Conscious sedation: an alternative to general anesthesia. J Dent Res. 1984;63:832-3.

5. Hempenstall PD, Campbell JP, Bajurnow AT, Reade PC, McGrath $\mathrm{B}$, Harrison LC. Cardiovascular, biochemical, and hormonal responses to intravenous sedation with local analgesia versus general anesthesia in patients undergoing oral surgery. J Oral Maxillofac Surg. 1986;44:441-6.

6. Corah NL. Development of a dental anxiety scale. J Dent Res. 1969;48:596.

7. Zacny JP, Hurst RJ, Graham L, Janiszewski DJ. Preoperative dental anxiety and mood changes during nitrous oxide inhalation. J Am Dent Assoc. 2002;133:82-8.

8. Eli I, Schwartz-Arad D, Baht R, Ben-Tuvim H. Effect of anxiety on the experience of pain in implant insertion. Clin Oral Implants Res. 2003;14:115-8.

9. Ellis S. Response to intravenous midazolam sedation in general dental practice. Br Dent J. 1996;180:417-20.

10. Parworth LP, Frost DE, Zuniga JR, Bennett T. Propofol and fentanyl compared with midazolam and fentanyl during third molar surgery. J Oral Maxillofac Surg. 1998;56:447-53.

11. Kunzelmann KH, Dünninger P. Dental fear and pain: effect on patient's perception of the dentist. Community Dent Oral Epidemiol. 1990;18:264-6.

12. Neverlien PO. Normative data for Corah's Dental Anxiety Scale (DAS) for the Norwegian adult population. Community Dent Oral Epidemiol. 1990;18:162.

13. Liddell A, Locker D. Gender and age differences in attitudes to dental pain and dental control. Community Dent Oral Epidemiol. 1997;25:314-8

14. Hägglin C, Berggren U, Hakeberg M, Hällstrom T, Bengtsson C. Variations in dental anxiety among middle-aged and elderly women in Sweden: a longitudinal study between 1968 and 1996. J Dent Res. 1999;78:1655-61.

15. Liau FL, Kok SH, Lee JJ, Kuo RC, Hwang CR, Yang PJ, et al. Cardiovascular influence of dental anxiety during local anesthesia for tooth extraction. Oral Surg Oral Med Oral Pathol Oral Radiol Endod. 2008;105:16-26.

16. Hashem AA, Claffey NM, O'Connell B. Pain and anxiety following the placement of dental implants. Int J Oral Maxillofac Implants. 2006;21:943-50

17. Frazer M, Hampson S. Some personality factors related to dental anxiety and fear of pain. Br Dent J. 1988;165:436-9.

18. Ganzberg S, Pape RA, Beck FM. Remifentanil for use during conscious sedation in outpatient oral surgery. J Oral Maxillofac Surg. 2002;60:244-50.

19. Garip H, Gürkan Y, Toker K, Göker K. A comparison of midazolam and midazolam with remifentanil for patient-controlled sedation during operations on third molars. Br J Oral Maxillofac Surg. 2007:45:212-6 